\title{
Inflammatory cells and cellular activation in the lower respiratory tract in Churg-Strauss syndrome
}

\author{
Armin Schnabel, Elena Csernok, Jörg Braun, Wolfgang L Gross
}

\begin{abstract}
Background-To obtain insight into the mechanisms of tissue injury in lung disease due to Churg-Strauss syndrome (CSS), the bronchoalveolar lavage (BAL) cell profile and the levels in the BAL fluid of cell products released by activated eosinophils and neutrophils were assessed.

Methods-Thirteen patients with active progressive CSS $(\mathbf{n}=7)$ or CSS in partial remission $(n=6)$ underwent clinical staging and bronchoalveolar lavage. The levels of eosinophil cationic protein (ECP), myeloperoxidase (MPO), and peroxidase activity in the BAL fluid were determined and the results were compared with those of 19 patients with pulmonary active Wegener's granulomatosis (WG) and nine
\end{abstract} control subjects.

Results-In patients with progressive CSS the BAL cell profile was dominated by eosinophils, neutrophil elevation being the exception. The eosinophilia was associated with high ECP levels $(4.39 \mathrm{ng} / \mathrm{ml}$ and $0.40 \mathrm{ng} / \mathrm{ml}$ in the two CSS groups compared with unmeasurable values in the controls). Individual patients with highly active CSS also had raised MPO levels, comparable to the levels in the most active WG patients. Peroxidase activity in the BAL fluid was $1.26 \mathrm{U} / \mathrm{ml}$ and $0.10 \mathrm{U} / \mathrm{ml}$ in the two groups of patients with CSS and $0.20 \mathrm{U} / \mathrm{ml}$ in the controls. Pulmonary disease in patients with WG was characterised by an extensive increase in MPO $(0.30$ $\mathrm{ng} / \mathrm{ml}$ versus $0.13 \mathrm{ng} / \mathrm{ml}$ in the controls) together with high peroxidase activity in the BAL fluid $(4.37 \mathrm{U} / \mathrm{ml})$, but only a small increase in ECP levels was seen. No correlation was found between the ECP and MPO levels in patients with CSS which suggests that eosinophil and neutrophil activation vary independently of each other.

Conclusions-These findings suggest that, in addition to eosinophil activation, neutrophil activation is an important feature in some patients with highly active CSS. The balance of neutrophil and eosinophil involvement appears to be variable and this may be one explanation for the individually variable treatment requirements of patients with CSS. (Thorax 1999;54:771-778)

Keywords: Churg-Strauss syndrome; eosinophils; neutrophils; bronchoalveolar lavage
Churg-Strauss syndrome (CSS) is a primary systemic vasculitis of mainly small and intermediate vessels with a propensity for lung involvement. ${ }^{12}$ It is commonly thought of as an autoimmune disease, ${ }^{3}$ but the mechanisms involved in the tissue injury are poorly understood. Prominent eosinophilia is one of the defining features of CSS. ${ }^{14}$ Its magnitude commonly reflects clinical disease activity and in many situations eosinophil suppression results in clinical improvement. ${ }^{125}$ Eosinophils were also found to be increased in the bronchoalveolar lavage (BAL) fluid of patients with pulmonary active CSS. ${ }^{6-8}$ Eosinophils can cause tissue injury by releasing a spectrum of toxic products. Eosinophil degranulation was found to occur at sites of disease in various hypereosinophilic disorders, ${ }^{9}$ but data to this effect from patients with CSS are few and inconsistent. $^{67}$

Moreover, it is unclear to what extent neutrophils are involved in lung disease due to CSS. Neutrophil activation is a central part of the pathogenesis of Wegener's granulomatosis (WG) and microscopic polyangiitis (MPA), which together with CSS make up the ANCA associated primary systemic vasculitides. ${ }^{3}{ }^{10}$ Abundant neutrophils and extracellular neutrophil products are present in florid tissue lesions due to WG and MPA and are thought to contribute to inflammatory tissue injury. ${ }^{11-13}$ Neutrophils can also be raised in the BAL fluid of patients with $\mathrm{CSS}^{7}$ and are present in active tissue lesions, although in fewer numbers than eosinophils. ${ }^{4}$ However, no data are available to enable us to estimate the magnitude of neutrophil activation and the balance of neutrophil and eosinophil activity in patients with CSS related lung disease.

This study was therefore undertaken to examine the BAL cell profile and markers of cellular activation in the BAL fluid of 13 consecutive patients with pulmonary active CSS. The level of eosinophil cationic protein (ECP) in BAL fluid was measured as a marker of eosinophil degranulation and myeloperoxidase (MPO) as a marker of neutrophil degranulation. The oxidant burden in the lower respiratory tract was estimated by measuring peroxidase activity. The findings in patients with CSS were compared with those from patients with pulmonary active WG.

\section{Methods}

PATIENTS

The CSS group comprised nine women and four men with a median age of 55 years (range 41-57) and a diagnosis of CSS according to the 1990 American College of Rheumatology
7 June 1999

Accepted for publication

11 June 1999 
Table 1 Characteristics of patients with progressive Churg-Strauss syndrome $(n=7)$ and Churg-Strauss syndrome in partial remission $(n=6)$

\begin{tabular}{lll}
\hline & Progressive disease & Partial remission \\
\hline Female:male & $6: 1$ & $3: 3$ \\
Age (years) & $50(39-55)$ & $57(42-60)$ \\
Duration of vasculitis (months) & $6(1-104)$ & $43(26-67)$ \\
ESR (mm/h) & $28(16-72)$ & $14(3-16)$ \\
C-reactive protein (mg/dl) & $1.7(0.6-11.7)$ & $0.5(0.4-0.7)$ \\
Blood eosinophil count (cells/ $\mu \mathrm{l})$ & $1470(550-2560)$ & $170(100-210)$ \\
Disease extent index (DEI score) & $5(3-9)$ & $4(4-6)$ \\
Cumulative organ involvement (n) & & 6 \\
Upper respiratory tract & 7 & 4 \\
Arthritis & 3 & 4 \\
Polyneuropathy & 4 & 1 \\
Kidney & 0 & 3 \\
Skin & 4 & 3 \\
Heart & 3 & 2 \\
Gastrointestinal & 1 & 1 \\
Myositis & 0 & $92(67-100)$ \\
VCIN (\% predicted) & $97(78-102)$ & $67(43-70)$ \\
FEV $/$ VCIN (\%) & $70(67-75)$ & $84(77-87)$ \\
TLCO/VA (\% predicted) & $88(71-92)$ & \\
\hline
\end{tabular}

VCIN = inspiratory vital capacity; $\mathrm{FEV}_{1}=$ forced expiratory volume in one second; TLCO = carbon monoxide transfer factor; $\mathrm{VA}=$ alveolar volume.

Values are median (interquartile range).

criteria and the 1992 Chapel-Hill definition (table 1). ${ }^{14}{ }^{15}$ All 13 patients had active pulmonary disease which manifested itself as bronchial asthma in eight patients and obstructive bronchitis in association with a history of bronchial asthma in five. Three patients also had parenchymal infiltrates at the time of examination. Staging investigations included lung function testing, chest radiography, bronchoscopy, BAL, echocardiography, otorhinolaryngological, ophthalmological and neurological consultation, cranial magnetic resonance imaging, and laboratory screening for anti-neutrophil cytoplasmic antibodies. ${ }^{16}$ Extrapulmonary organ manifestations were chronic rhinitis or sinusitis in all 13 patients, cutaneous leukocytoclastic vasculitis or nodular skin infiltrates in seven, peripheral neuropathy mainly in the form of mononeuritis multiplex in eight, cardiac involvement in the form of dilatory cardiomyopathy, ventricular or supraventricular arrhythmias or pericardial effusion in six, arthritis in seven, intestinal involvement in the form of diarrhoea or abdominal cramping which resolved upon immunosuppression in three, and glomerulonephritis and myositis in one patient each (table 1). The duration of vasculitic disease ranged from one to 111 months with a mean of 28 months. The time span from the onset of asthma to generalised disease was 1-112 months with a median of 23 months. Histological confirmation was obtained in the upper and lower respiratory tract in five patients and at various other sites in the remaining eight. The patients were subdivided according to disease status into a group of seven patients with highly active progressive disease and a group of six patients with CSS in partial remission. At the time of examination all patients in the former group were on prednisolone at a median dose of $10 \mathrm{mg} /$ day. One of these patients experienced a major relapse while he was also receiving low dose methotrexate and another patient relapsed while she was on cyclophosphamide. Five of the six patients in partial remission were receiving a median dose of $12 \mathrm{mg} /$ day pred- nisolone and two patients were also on cyclophosphamide.

The WG group comprised 19 patients diagnosed according to the 1990 American College of Rheumatology criteria and the 1992 ChapelHill definition (table 2). ${ }^{15}$ All patients had active progressive disease. In 12 patients histological confirmation was obtained from the upper and lower respiratory tracts, and from various other sites in the remaining patients. Pulmonary disease manifested itself radiologically in the form of ill defined infiltrates or consolidation in 12 patients and solitary or multiple nodules in five. Two patients had ulcerative and granulomatous bronchitis in association with a lymphocytic BAL profile. Extrapulmonary organ involvement included the upper respiratory tract in all 19 patients, the kidneys in 14 patients, the eyes in 12 , the peripheral nervous system in seven, the skin in four, the heart in three, the gastrointestinal tract in two, the central nervous system in two, and the musculoskeletal system in 16 patients. At the time of examination 12 patients were not receiving any immunosuppressive medication; in the others a major relapse occurred while receiving low dose prednisolone $(n=2)$, low dose methotrexate $(n=2)$, cotrimoxazole $(n=$ $2)$, or cyclophosphamide $(n=1)$.

There were nine patients in the control group (five women) with a mean age of 33 years (interquartile range 29-39). Six of these had been transferred to our department for evaluation of suspected systemic rheumatic disease and turned out to be free of inflammatory systemic or pulmonary disease and three were healthy laboratory personnel. All nine subjects had a normal chest radiograph, normal lung function, and a normal BAL cell profile. All patients and the control subjects were non-smokers and were free of clinical or microbiological evidence of infection.

\section{BRONCHOSCOPY}

Fibreoptic bronchoscopy was performed after local anaesthesia with lidocaine and premedication with atropine and a morphine antitussive. The bronchoscope was wedged into a seg-

Table 2 Characteristics of patients with progressive Wegener's granulomatosis $(n=19)$

\begin{tabular}{ll}
\hline Female:male & $8: 11$ \\
\hline Age (years) & $60(54-64)$ \\
Duration of disease (months) & $39(11-87)$ \\
ESR (mm/h) & $90(40-105)$ \\
C-reactive protein (mg/dl) & $11.3(2.1-15.8)$ \\
Disease extent index (DEI score) & $7(3-11)$ \\
Cumulative organ involvement (n) & \\
$\quad$ Upper respiratory tract & 19 \\
Kidney & 14 \\
Eye & 12 \\
Arthritis & 16 \\
Polyneuropathy & 7 \\
Central nervous system & 2 \\
Skin & 4 \\
Heart & 3 \\
Gastrointestinal & 2 \\
VCIN (\% predicted) & $94(78-106)$ \\
FEV $/$ VCIN (\%) & $71(62-78)$ \\
TLCO/VA (\% predicted) & $115(95-120)$ \\
\end{tabular}

VCIN = inspiratory vital capacity; $\mathrm{FEV}_{1}=$ forced expiratory volume in one second; $\mathrm{TLCO}_{\mathrm{LCO}}$ carbon monoxide transfer factor; $\mathrm{VA}=$ alveolar volume.

Values are median (interquartile range). 
mental bronchus and 12 fractions of $20 \mathrm{ml}$ sterile $0.9 \%$ saline were instilled and aspirated. Fluid recovery was at least $60 \%$. The first two fractions were set apart and the third to twelfth fractions were pooled and further analysed. Aliquots of the material were examined for conventional bacterial pathogens, acid-fast bacteria, Legionella species, Chlamydia species, Mycoplasma species, and Pneumocystis carinii and any infected material was omitted from further study.

BAL CELL ANALYSIS

The lavage material was filtered through surgical gauze and centrifuged at $4^{\circ} \mathrm{C}$. The cell pellet was resuspended in RPMI 1640 medium (Gibco, Eggenstein, Germany) to a density of $10^{6}$ cells $/ \mathrm{ml}$. Cytospin preparations were prepared with a Shandon II cytocentrifuge (Shandon Products, Cheshire, UK) and stained using the May-Giemsa-Gruenwald stain. The cell differential was assessed microscopically by counting 400 cells. Normal reference values in this laboratory are $\leqslant 15 \%$ lymphocytes, $\leqslant 5 \%$ neutrophils, and $<1 \%$ eosinophils.

MEASUREMENT OF SOLUBLE CELL PRODUCTS IN BAL FLUID

The BAL fluid was concentrated by pressure filtration under nitrogen using Amicon YM10 membranes with a molecular weight cut off of 10 kD (Millipore Corporation, Eschborn, Germany). Filtration was stopped when the retained volume amounted to $1.2-1.5 \mathrm{ml}$, resulting in a mean concentration factor of 37 . Solute concentrations in the original BAL fluid were calculated by dividing the measured concentration by the individual concentration factor. The level of ECP in concentrated BAL fluid was measured using the Unicap ECPfluoroimmunoassay (Pharmacia, Freiburg, Germany) according to the recommendations of the manufacturer. The detection threshold in the BAL fluid concentrate was $2 \mathrm{ng} / \mathrm{ml}$. MPO was measured using the myeloperoxidase capture ELISA (Calbiochem-Novabiochem Corporation, San Diego, California, USA) according to the recommendations of the manufacturer. The threshold of detection in the BAL fluid concentrate was $1.2 \mathrm{ng} / \mathrm{ml}$. Peroxidase activity was measured using the K-blue assay as described by Segelmark et al. ${ }^{18}$ The tetramethylbenzidine and hydrogen peroxidase containing substrate/chromogen mixture was purchased from Neogen, Lexington, Kentucky, USA. Briefly, $50 \mu \mathrm{l}$ of concentrated BAL fluid (1:2 diluted in PBS) was mixed with $50 \mu$ of the substrate solution and the absorbance was measured at $660 \mathrm{~nm}$ after 30 minutes. Measurement of progressive dilutions of specimens of BAL fluid gave values which parallelled the standard curve, thus verifying the specificity of the method. Moreover, purified myeloperoxidase purchased from Calbiochem Corporation (Bad Soden, Germany) was diluted serially and measured using the K-blue assay and the aforementioned capture ELISA. This gave a close correlation between peroxidase activity and MPO measurements. The detection threshold of the peroxidase assay was $1.5 \mathrm{ng} / \mathrm{ml}$. All assays were run in duplicate.

ANALYSIS OF DATA

Data are presented as median with the 25th and 75 th percentiles. Values for the BAL cell profile are expressed as percentage of total BAL cells. Concentrations of BAL fluid solutes are presented as concentrations in the original lavage fluid. Differences between the groups were tested for statistical significance by the Kruskal-Wallis test, followed by the MannWhitney $U$ test for pairwise differences. The Spearman rank test was used to calculate correlations between cell numbers and solute concentrations.

\section{Results}

BAL CELL PROFILE

An abnormal BAL cell profile was found in 11 of the 13 patients with CSS. The most prominent abnormality was an excess of eosinophils (fig 1A). The median eosinophil count was $34 \%$ (interquartile range $8-71 \%$ ) in the patients with progressive disease and $0.5 \%$ (interquartile range $0-5 \%$ ) in the patients in partial remission, compared with a normal reference value of $<1 \%$. The difference in the eosinophil count between the two CSS subgroups was statistically significant $(p=0.015)$. The eosinophil count in the BAL fluid proved to be more sensitive in reflecting disease activity than the eosinophil count in the blood, with three patients (one in the high activity group and two in the group in partial remission) having increased levels of eosinophils in the BAL fluid in the presence of normal blood eosinophils. Increased lymphocyte levels were found in two patients in the group with highly active disease and in two of those with CSS in partial remission, but only one patient in the former group had raised BAL neutrophils.

Thirteen of the 19 patients with highly active WG had an abnormal BAL cell profile (fig 1B). On the basis of the BAL cell pattern these patients fell into three categories. Seven patients had raised neutrophil levels (range $6-18 \%$, normal reference value $\leqslant 5 \%$ ), six had raised lymphocyte levels (interquartile range $18-42 \%$, normal reference value $\leqslant 15 \%$ ), and the remaining six patients had a normal BAL cell profile. Low grade elevation of the eosinophils in the range of $1-3 \%$ was found in four patients.

CELLULAR ACTIVATION MARKERS IN BAL FLUID All CSS patients with highly active disease and five of the six patients in partial remission had measurable ECP levels in the BAL fluid whereas none of the controls had a measurable ECP level (fig 2). The median ECP level was $4.39 \mathrm{ng} / \mathrm{ml}$ (interquartile range 4.00-6.50) in the highly active patients and this was significantly higher than the level of $0.40 \mathrm{ng} / \mathrm{ml}$ (interquartile range $0-0.56$ ) in the patients in partial remission $(p=0.004)$. A statistically significant correlation was found between the ECP level in the BAL fluid and the percentage eosinophil count in the BAL fluid of the 13 patients with CSS $(R=0.774, \mathrm{p}=0.002$; fig 3$)$, 

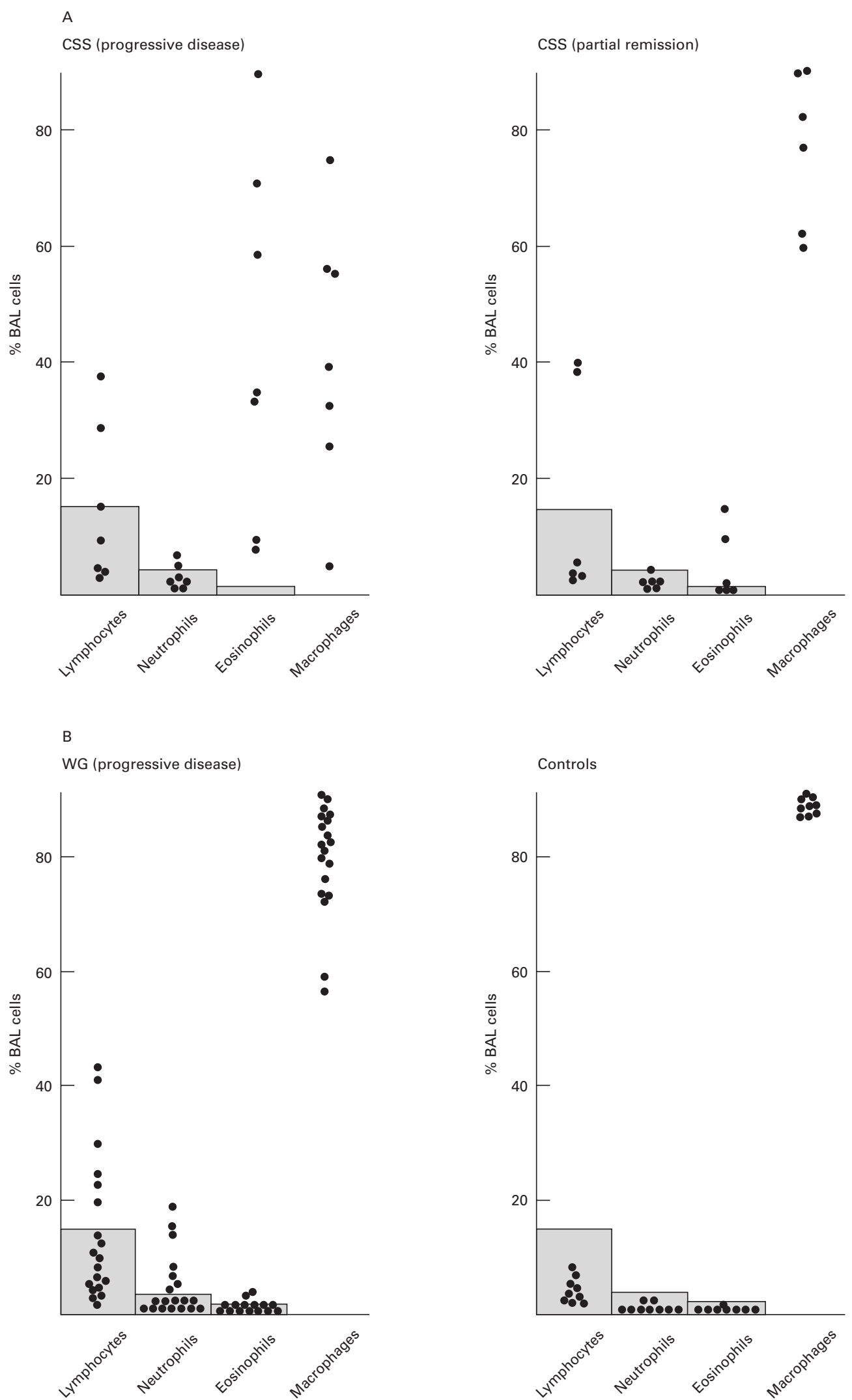

Figure 1 BAL cell profiles in (A) patients with progressive Churg-Strauss syndrome (CSS) and CSS in partial remission and (B) patients with progressive Wegener's granulomatosis (WG) and controls. Shaded areas represent normal reference values.

and the strength of the correlation was very similar when absolute counts of BAL eosinophils were used in this analysis $(R=0.724, \mathrm{p}$
$=0.005)$. This suggests that the BAL eosinophils were the dominant source of the ECP in the BAL fluid. MPO levels in the BAL fluid 


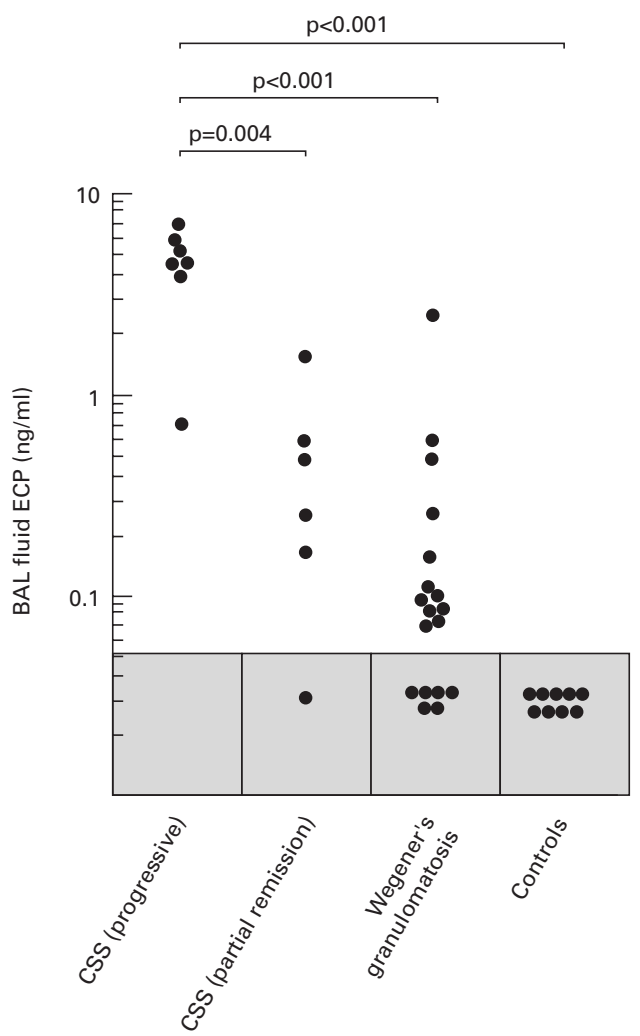

Figure 2 Eosinophil cationic protein (ECP) levels in $B A L$ fluid in patients with Churg-Strauss syndrome, Wegener's granulomatosis, and controls. Shaded area marks values below the detection threshold.

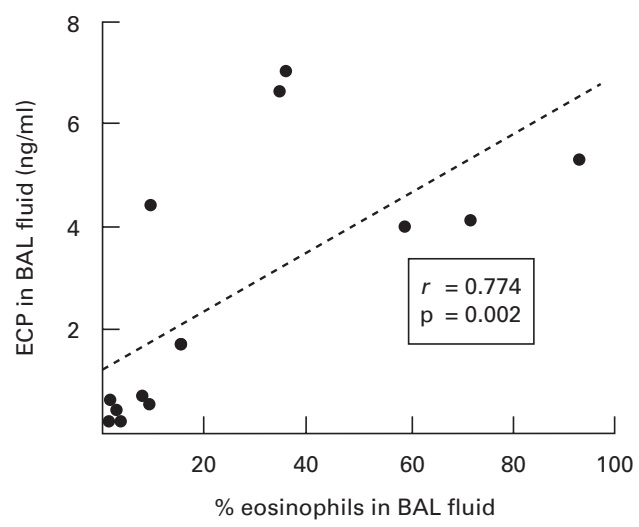

Figure 3 Correlation between the level of eosinophil cationic protein (ECP) and the eosinophil count in BAL fluid in patients with Churg-Strauss syndrome.

spanned essentially the same range in the patients with highly active CSS as in the patients with WG (fig 4), resulting in virtually identical median levels in the two groups (0.32 ng/ml and $0.30 \mathrm{ng} / \mathrm{ml}$, respectively). The MPO levels in the patients with WG differed significantly from the controls $(p=0.018)$, but the number of cases with progressive CSS was too small for the increase in MPO levels to reach statistical significance. No correlation was found between the ECP and MPO levels in the BAL fluid of patients with either CSS or WG.

The patients with highly active WG had moderately increased ECP levels in the BAL fluid which were significantly higher than those in the controls $(p=0.019)$ but lower than those

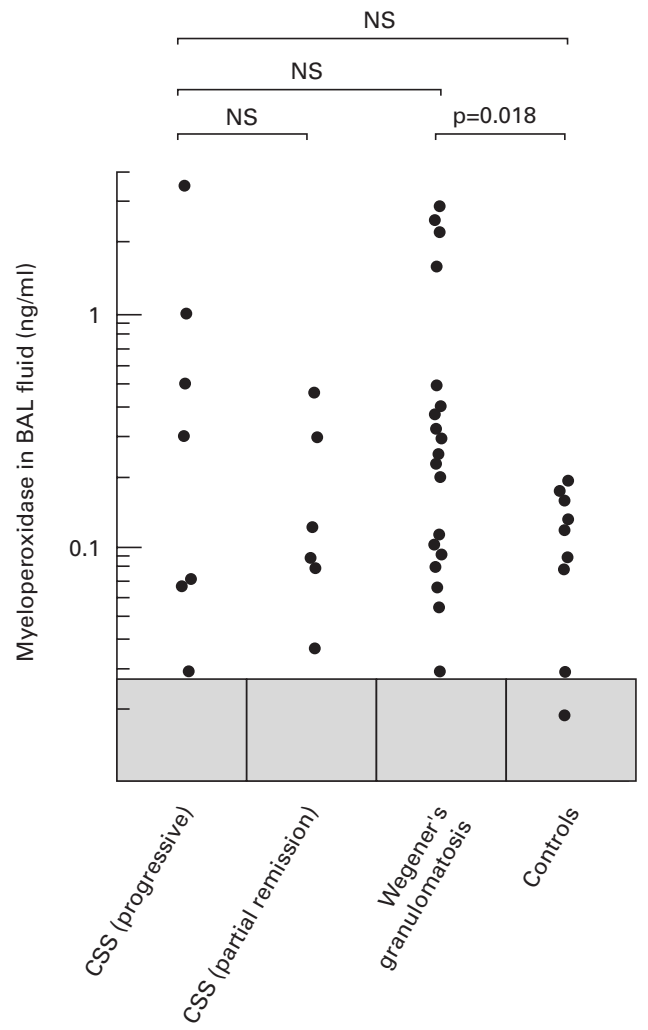

Figure 4 Myeloperoxidase (MPO) levels in the BAL fluid of patients with Churg-Strauss syndrome, Wegener's granulomatosis, and controls. NS = not statistically significant.

in the patients with highly active CSS $(0.07 \mathrm{ng} /$ $\mathrm{ml}$ versus $4.39 \mathrm{ng} / \mathrm{ml}, \mathrm{p}<0.001$; fig 2 ). The median MPO level in the BAL fluid of patients with WG was significantly higher than the median MPO level of the controls ( $p=0.018$; fig 4). The highest MPO levels were seen in the patients with an increased BAL neutrophil count. The patients with a lymphocytic pattern had somewhat lower levels, while patients with a normal BAL cell profile also had normal MPO levels $(1.40 \mathrm{ng} / \mathrm{ml}, 0.30 \mathrm{ng} / \mathrm{ml}$, and $0.17 \mathrm{ng} / \mathrm{ml}$ in the WG patients compared with $0.08 \mathrm{ng} / \mathrm{ml}$ in the controls). The coefficient of correlation between the MPO level in the BAL fluid and the percentage of BAL neutrophils in these patients was $R=0.610(\mathrm{p}=0.005)$, and the coefficient of correlation between the MPO level and the absolute BAL neutrophil count was $R=0.539(\mathrm{p}=0.021)$.

The median peroxidase levels in the BAL fluid of patients with progressive CSS were $1.26 \mathrm{U} / \mathrm{ml}$ (interquartile range $0.26-3.14 \mathrm{U} /$ $\mathrm{ml}$ ) which were significantly higher than those in the patients with CSS in partial remission (median $0.10 \mathrm{U} / \mathrm{ml}$; interquartile range $0-0.29 ; \mathrm{p}=0.021$ ) and the controls (median $0.20 \mathrm{U} / \mathrm{ml}$, interquartile range $0-0.37 ; \mathrm{p}=$ 0.030 ; fig 5). A statistically significant correlation between the peroxidase and ECP levels in the BAL fluid of the 13 patients with CSS $(R=$ $0.674, p=0.011$ ) suggests that the eosinophils were a major source of the excess peroxidase. In the patients with WG a correlation was found between peroxidase levels and the percentage neutrophil count in the BAL fluid 


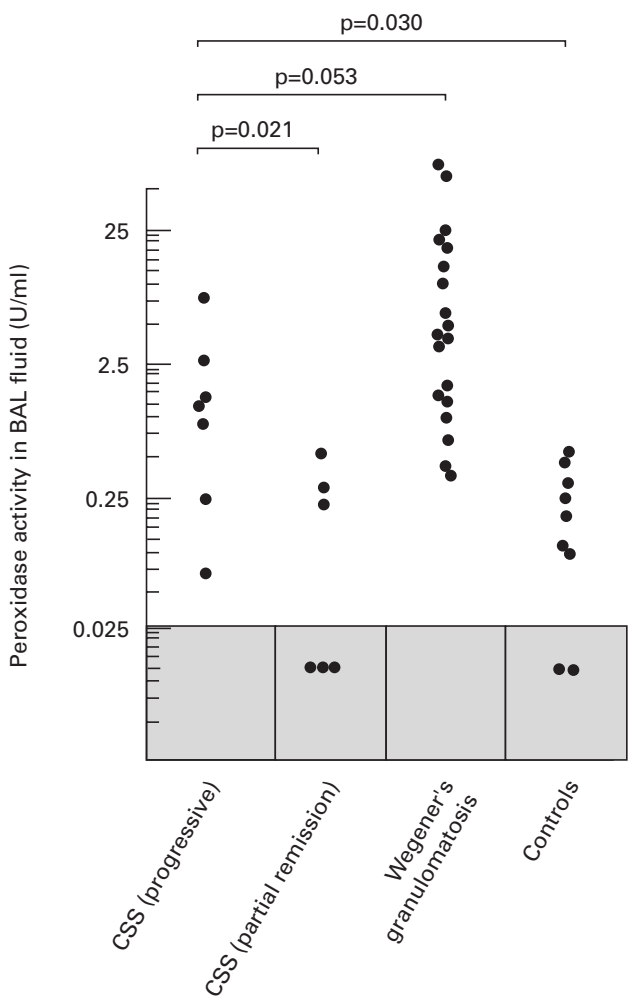

Figure 5 Peroxidase activity in BAL fluid in patients with Churg-Strauss syndrome, Wegener's granulomatosis, and controls.

$(R=0.571, \mathrm{p}=0.011)$ and between peroxidase levels and the absolute neutrophil count $(R=$ $0.531, \mathrm{p}=0.019$ ), but no correlation was found between peroxidase levels and the eosinophil count. This suggests that, in patients with WG, the neutrophils rather than the eosinophils are an important source of the excess peroxidase activity.

\section{Discussion}

Previous studies of the pulmonary pathology of the small vessel vasculitides have relied mainly on materials obtained by open or transbronchial lung biopsy or necropsy. ${ }^{41}$ Small vessel vasculitides are characterised by a patchy distribution of the tissue lesions ${ }^{411}$ and, indeed, biopsy studies in patients with WG and CSS have shown that the small specimens obtained by forceps biopsy are very heterogeneous with respect to the presence of vasculitis, granuloma, necrosis, and non-specific changes. ${ }^{19}$ This entails the risk of a large sampling error for transbronchial biopsy. In contrast to this, BAL extracts materials from a much larger tissue volume and is predictably less susceptible to the problem of inadequate sampling. Moreover, by yielding not only cellular material but also extracellular solutes, BAL materials can provide functional information not readily obtained by morphologic analysis. Based on these considerations we employed BAL rather than transbronchial biopsy to study the involvement of eosinophils and neutrophils in lower respiratory tract disease due to CSS and WG.

In agreement with previous studies, ${ }^{6-8}$ eosinophilia was the most common abnormality in the BAL cell profile in patients with CSS. No correlation was found between the eosinophil count in the BAL fluid and the blood, but the 10 patients with BAL eosinophilia included three who had a normal eosinophil count in the blood. That the BAL eosinophil count in pulmonary active disease can be a more sensitive indicator of disease than the cell count in blood was also suggested by the observations of Wallaert et al who found that corticosteroid treatment can normalise the blood eosinophils in patients with CSS without having any major effect on the BAL eosinophil count. ${ }^{7} \mathrm{~A}$ borderline increase in the BAL neutrophils was found in only two patients with CSS, both of whom had chronic obstructive airways disease that had evolved from longstanding bronchial asthma. The association of chronic obstructive airways disease with increased BAL neutrophils is well documented ${ }^{2021}$ and we believe that this also accounts for the BAL neutrophilia in these two patients.

ECP is a constituent of the specific granules of eosinophils, from which it is released upon cellular activation together with other compounds. ${ }^{9}$ Extracellular ECP has been detected at sites of active disease in several hypereosinophilic disorders including allergic bronchial asthma, the eosinophilic pneumoniae, idiopathic hypereosinophilic syndrome, and CSS. ${ }^{92}$ Increased ECP levels in the serum of patients with CSS were recently shown to vary with disease activity. ${ }^{23}$ High ECP levels have been detected in BAL fluid in patients with allergic bronchial asthma, ${ }^{2021}{ }^{24}$ idiopathic pulmonary fibrosis, ${ }^{25}$ chronic eosinophilic pneumonia and eosinophilic granuloma, ${ }^{26}$ and a quantitative correlation was found between the severity of allergic asthma and ECP levels. ${ }^{24}$ Based on these findings, the ECP level in biological fluids is used as a quantitative measure of eosinophil accumulation and activation and in the patients with CSS in this study they obviously reflect extensive local degranulation of the eosinophils. The correlation between ECP levels and the eosinophil count in the BAL fluid suggests that the eosinophils on the epithelial surfaces are an important, although not necessarily the exclusive, source of the ECP measured in the BAL fluid. High eosinophil counts were also associated with high peroxidase levels in the BAL fluid, and this activity correlated significantly with ECP levels. Peroxidase is a secretory product of both eosinophils and neutrophils ${ }^{927}$ but, given the aforementioned correlation, the main source of the peroxidase in these CSS patients appears to be the eosinophils.

MPO is a secretory product of neutrophils and macrophages ${ }^{27}{ }^{28}$ but, by all accounts, the amount of macrophage-derived MPO in inflammatory tissues is small. ${ }^{28}$ Extracellular MPO and other granule constituents are therefore useful indices of local degranulation of neutrophils. Pulmonary disorders involving a strong neutrophil component are associated with increased MPO levels in the BAL fluid. Prominent examples of this are bacterial infections, ${ }^{29}$ chronic bronchitis, ${ }^{20} 2130$ idiopathic pulmonary fibrosis, ${ }^{25}{ }^{31-33}$ and interstitial lung 
disease due to systemic sclerosis. ${ }^{32}$ Against this background the MPO levels in patients in the present study with highly active CSS reflect the local degranulation of substantial numbers of neutrophils. It was apparently the wide scatter of individual values and the limited number of cases which precluded a statistically significant difference between the median MPO levels of the CSS patients and the controls. However, individual patients in the highly active CSS group had MPO levels that equalled the levels in patients with the most active WG, suggesting that the magnitude of neutrophil activation in CSS can attain the same high level as in WG.

Neutrophil accumulation and release of neutrophil contents at sites of highly active disease are well known features of WG. ${ }^{12}{ }^{13}$ In agreement with previous reports, ${ }^{34}$ a predominantly neutrophilic BAL cell profile was found in the patients in this study with WG with florid pulmonary disease which manifested itself radiologically as infiltrates or extensive consolidation, whereas nodular lesions were more common in the patients with a lymphocytic or normal cell profile. Patients with increased BAL neutrophils did, indeed, have the highest MPO levels in the BAL fluid but raised levels were also found in the patients with increased BAL lymphocytes but normal neutrophils. This suggests that the MPO recovered in the BAL fluid originates not only from cells present on the epithelial surfaces but also from neutrophils located in the tissue. This would also explain the finding that several of the patients with highly active CSS had high MPO levels in the BAL fluid in the absence of increased BAL neutrophils.

Most of the patients with highly active CSS therefore had BAL fluid findings that suggest concomitant eosinophil and neutrophil activation, but the absence of a correlation between the BAL fluid levels of ECP and MPO implies that the magnitude of eosinophil and neutrophil activation was not interrelated. At one end of a wide spectrum of findings was patient 7 who underwent BAL during a major relapse with exacerbation of obstructive lung disease, rapid deterioration of polyneuropathy, scleritis, and profound malaise. While indices of eosinophil involvement stayed fairly low (blood eosinophils $1400 / \mu$, BAL eosinophils $8 \%$, ECP in the BAL fluid $4.4 \mathrm{ng} / \mathrm{ml})$, the MPO $(3.28 \mathrm{ng} /$ $\mathrm{ml}$ ) and peroxidase levels $(3.14 \mathrm{U} / \mathrm{ml})$ approached those of the most active WG patients, reflecting extensive neutrophil activation. An example from the other end of the spectrum is patient 1 who had excessive eosinophil stimulation (BAL eosinophils $92 \%$, ECP in the BAL fluid $5.2 \mathrm{ng} / \mathrm{ml}$ ) together with a low normal neutrophil count and only trace MPO levels in the BAL fluid. In contrast to patient 7, whose relapse could only be controlled with daily oral cyclophosphamide over the next several months, patient 1 achieved prompt remission of her pleuritic and interstitial lung disease with short term treatment with prednisolone and subsequent interferon- $\alpha$.

Taken together, the present findings enhance the view that tissue injury by eosinophil products is an important disease mechanism in
CSS. Indeed, an increase in the ECP levels in the BAL fluid, which reflects eosinophil degranulation, was the most consistent BAL finding in these patients. Neutrophil degranulation appears to be a further mechanism involved in this disorder, but the present results suggest that this is less consistent than eosinophil degranulation. These findings suggest also that the magnitude of neutrophil and eosinophil degranulation vary independently of each other. Treatment requirements of CSS range from moderate corticosteroid doses in some patients to intense immunosuppression in others. It is tempting to speculate that the different responsiveness of eosinophils and neutrophils to therapeutic agents is one reason for the heterogeneity of the clinical responses, but this needs to be examined in studies designed specifically for this purpose.

Supported by the Bundesminister für Bildung und Forschung, grant no. 01 VM 9306.

1 Lanham JG, Elkon KB, Pusey CD, et al. Systemic vasculitis with asthma and eosinophilia: a clinical aproach to the Churg-Strauss syndrome. Medicine 1984;63:65-81.

2 Guillevin L, Cohen P, Gayraud M, et al. Churg-Strauss syndrome. Clinical study and long-term follow-up of 96 patients. Medicine 1999;78:26-37.

3 Gross WL, Schmitt WH, Csernok E. ANCA and associated diseases: immunodiagnostic and pathogenetic aspects. Clin Exp Immunol 1993;91:1-12.

4 Churg J. Churg-Strauss syndrome. In: Thurlbeck WM, Churg AM, eds. Pathology of the lung. Stuttgart, New York: Thieme, 1995: 425-35.

5 Tatsis E, Schnabel A, Gross WL. Interferon-alpha treatment of four patients with the Churg-Strauss syndrome. Ann Intern Med 1998;129:370-4.

6 Olivieri D, Pesci A, Bertorelli G. Eosinophilic alveolitis in immunologic interstitial lung disorders. Lung 1990;Suppl: 964-73.

7 Wallaert B, Gosset P, Prin L, et al. Bronchoalveolar lavage in allergic granulomatosis and angiitis. Eur Respir $\mathcal{F}$ 1993;6: 413-7.

8 Schmidt B, Teschler H, Kroegel C. Bronchoalveolar lavage cell profiles in Wegener's granulomatosis, chronic eosinophilic pneumonia and Churg-Strauss syndrome. Eur Respir F 1989;2(Suppl 8):641S

9 Rothenberg ME. Eosinophilia. N Engl f Med 1998;338: 1592-600.

10 Jennette JC, Falk RJ. Pathogenesis of the vascular and glomerular damage in ANCA-positive vasculitis. Nephrol glomerular damage in ANCA-positive
Dial Transplant 1998;13(Suppl 1):16-20.

11 Travis WD, Hoffman GS, Leavitt RY, et al. Surgical pathology of the lung in Wegener's granulomatosis. Am f Pathol 1991;15:315-33.

12 Brouwer E, Huitema M, Mulder AHL, et al. Neutrophil activation in vitro and in vivo in Wegener's granulomatosis. Kidney Int 1994;45:1120-31.

13 Mrowka C, Csernok E, Gross WL, et al. Distribution of the granulocyte serine proteinases proteinase 3 and elastase in human glomerulonephritis. Am f Kidney Dis 1995;25:25361.

14 Masi AT, Hunder GG, Lie JT, et al. The American College of Rheumatology 1990 criteria for the classification of Churg-Strauss syndrome (allergic granulomatosis and angiitis). Arthritis Rheum 1990;33:1094-100.

15 Jennette JC, Falk RJ, Andrassy K, et al. Nomenclature of systemic vasculitis. Proposal of an International Consensus Conference. Arthritis Rheum 1994;37:187-92.

16 Nölle B, Specks U, Lüdemann J, et al. Anticytoplasmic autoantibodies: their immunodiagnostic value in Wegener's granulomatosis. Ann Intern Med 1989;111:28-40.

17 Leavitt RY, Fauci AS, Bloch DA, et al. The American College of Rheumatology 1990 criteria for the classification of Wegener's granulomatosis. Arthritis Rheum 1990;33: $1101-7$

18 Segelmark M, Persson B, Hellmark T, et al. Binding and inhibition of myeloperoxidase (MPO): a major function of ceruloplasmin? Clin Exp Immunol 1997;108:167-74.

19 Schnabel A, Holl-Ullrich K, Dalhoff K, et al. Efficacy of transbronchial biopsy in pulmonary vasculitides. Eur Respir f 1997; 10:2738-43.

20 Lacoste JY, Bousquet J, Chanez P, et al. Eosinophilic and neutrophilic inflammation in asthma, chronic bronchitis, and chronic obstructive disease. $\mathcal{F}$ Allergy Clin Immunol 1993;92:537-48.

21 Chanez P, Enander I, Jones I, et al. Interleukin 8 in bronchoalveolar lavage of asthmatic and chronic bronchitis

22 Tai P, Holt ME, Denny P, et al. Deposition of eosinophil cationic protein in granulomas in allergic granulomatosis
and vasculitis: the Churg-Strauss syndrome. BMF 1984; 289:400-2. 
23 Schmitt WH, Csernok E, Kobayashi S, et al. Churg-Strauss syndrome: serum markers of lymphocyte activation and syndrome: serum markers of lymphocyte activation

24 Bousquet J, Chanez P, Lacoste JY, et al. Indirect evidence of bronchial inflammation assessed by titration tory mediators in BAL fluid of patients with asthma. $\mathcal{F}$ Allergy Clin Immunol 1991;88:649-60.

25 Hällgren R, Bjermer R, Lundgren R, et al. The eosinophil component of the alveolitis in idiopathic pulmonary fibrosis. Am Rev Respir Dis 1989;139:373-7.

26 Shijubo N, Shigehara K, Hirasawa M, et al. Eosinophilic cationic protein in chronic eosinophilic pneumonia and eosinophilic granuloma. Chest 1994;106:1481-6.

27 Borregaard N, Cowland JB. Granules of the human polymorphonuclear leukocyte. Blood 1997;10:3503-21.

28 Schmekel B, Karlsson SE, Linden M, et al. Myeloperoxidase in human lung lavage. I. A marker of local neutrophil activity. Inflammation 1990;14:447-54

29 Braun J, Dalhoff K, Lipp R, et al. Myeloperoxidase, Lactoferrin und Elastase in bronchoalveolärer Lavage und ferrin und Elastase in bronchoalveolärer Lavage und
Plasma bei Pneumonie. Pneumologie 1992;46:141-7.
30 Riise G, Ahlstedt S, Larsson S, et al. Bronchial inflammation in chronic bronchitis assessed by measurement of cell products in bronchial lavage fluid. Thorax 1995;50:360-5. 31 Pohl WR, Schenk E, Umek H, et al. Aussagekraft der Sekretionsprodukte von Eosinophilen und Neutrophilen in der bronchoalveolären Lavage bei Patienten mit idiopathischer Lungenfibrose. Wien Klin Wochenschr 1993;105:387-92.

32 Cailes JB, O'Connor C, Pantelidis P, et al. Neutrophil activation in fibrosing alveolitis: a comparison of lone cryptogenic fibrosing alveolitis and systemic sclerosis. Eur Respir f 1996;9:992-9.

33 Cantin AM, North SL, Fells GA, et al. Oxidant-mediated epithelial cell injury in idiopathic pulmonary fibrosis. $7 \mathrm{Clin}$ Invest 1987;79:1665-73.

34 Hoffman GS, Sechler JMG, Gallin JI, et al. Bronchoalveolar lavage analysis in Wegener's granulomatosis. Am Rev Respir Dis 1991;143:401-7.

35 Schnabel A, Reuter M, Gloeckner K, et al. Bronchoalveolar lavage cell profiles in Wegener's granulomatosis. Respir Med 1999 (in press). 